\title{
Nicht entleihbar
}

\section{KERNFORSCHUNGSZENTRUM}

\section{KARLSRUHE}

Juli 1969

\section{Feb 10 an}

Institut für Kernverfahrenstechnik

Impulsradiolyse bei hohem Druck: Druckabhängigkeit des Absorptionsspektrums und der Lebensdauer hydratisierter Elektronen

Anordnung zur Impulsradiolyse bei hohem Druck

U. Schindewolf, H. Kohrmann, G. Lang

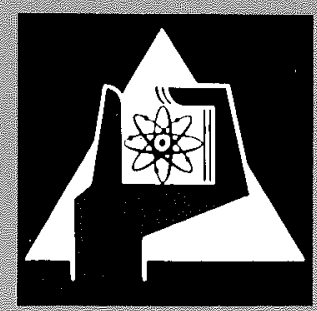




\section{SONDERDRUCK}

Impulsradiolyse bei hohem Druck:

Druckabhängigkeit des Absorptionsspektrums und der Lebensdauer hydratisierter Elektronen $\left.{ }^{[*} *\right]$

\section{Von U. Schindewolf, H. Kohrmam und G. Lang [*]}

Physikalische und chemische Eigenschaften solvatisierter Elektronen in flüssigem Ammoniak [1] (elektrische Leitfähigkeit [2], Absorptions- [3,4], Reflexions- [4] und ESR-Spektrum [5], physikalische [6] und chemische Gleichgewichte [7]) hängen stark vom Druck ab. Die in Metall-AmmoniakLösungen durch Dissoziation des Metalls entstehenden solvatisierten Elektronen reagieren nur langsam mit dem Lösungsmittel und haben eine hohe Lebensdauer ( $>$ Monate).

Vergleichende Untersuchungen des Druckeinflusses an hydratisierten, d.h. in Wasser gelösten Elektronen ${ }^{[8]}$ setzen wegen deren schneller Reaktion mit Wasser (Lebensdauer $<1 \mathrm{~ms}$ ) die Erzeugung dieser Elektronen unter Druck und schnell ansprechende Nachweismethoden voraus.

Wir haben nun die Druckabhängigkeit des Absorptionsspektrums und der Lebensdauer hydratisierter Elektronen gemessen, die durch Radiolyse wäßriger Lösungen mit den energiereichen Elektronen eines im Impulsbetrieb arbeitenden Elektronenlinearbeschleunigers gebildet wurden (bis zu 300 Impulse von $5 \mu$ s Dauer je s; $15 \mathrm{MeV}, 0.5 \mathrm{~A}$ ). Die Bestrahlungen wurden in einer Hochdruckküvette [9] (Wandstärke $2.5 \mathrm{~mm}$ ) im gekreuzten Strahlengang des Beschleunigers und eines Spektralphotometers mit Photokathode als Lichtdetektor durchgeführt.

Durch strahleninduzierte Ionisation des Wassers entstehen hydratisierte Elektronen,

$\mathrm{H}_{2} \mathrm{O} \rightarrow \mathrm{H}_{2} \mathrm{O}^{+}+\mathrm{e}^{-}$

die mit zugesetzten Substraten, mit anderen Radiolyseprodukten $\left(\mathrm{H}^{+}, \mathrm{OH}, \mathrm{H}\right.$ etc.) sowie mit dem Wasser selbst reagieren. Dic "bimolekulare" Reaktion mit Wasser führt zu Wasserstoff $\left(k \approx 10^{10} 1 / \mathrm{mol}^{-\mathrm{s}}\right)^{[8]}$

$2 \mathrm{e}^{-}+2 \mathrm{H}_{2} \mathrm{O} \rightarrow \mathrm{H}_{2}+2 \mathrm{OH}^{-}$

und begrenzt bei Abwesenheit anderer reaktiver Stoffe dic Lebensdauer der Elektronen. Um nur diese Reaktion beobachten zu können, werden die anderen Radiolyseprodukte durch Wasserstoff und Hydroxidionen abgefangen [8] (Vorratslösung: $0.01 \mathrm{M} \mathrm{NaOH}$, unter Normaldruck mit Wasserstoff gesättigt):

$\mathrm{H}_{2} \mathrm{O}^{+} \rightarrow \mathrm{H}^{+}+\mathrm{OH}$

$\mathrm{H}^{+}+\mathrm{OH}^{-} \rightarrow \mathrm{H}_{2} \mathrm{O}$

$\mathrm{OH}+\mathrm{H}_{2} \rightarrow \mathrm{H}_{2} \mathrm{O}+\mathrm{H}$

$\mathrm{H}+\mathrm{OH}^{-} \rightarrow \mathrm{H}_{2} \mathrm{O}+\mathrm{e}^{-}$

Durch Reaktion (6) wird so zugleich dic Ausbeute an hydratisierten Elektronen verdoppelt. Da sich die Elektronen dann - sofern keine Verunreinigungen zugegen sind - nur mit Wasser nach Reaktion (2) umsetzen und sich die Reaktionsfolge (1) bis (6) kompensiert, erfährt die Lösung bei der Bestrahlung keine bleibende Veränderung.

Nach einem Elektronenimpuls nimmt die Lichtdurchlässigkeit der Lösung im Wellenlängenbereich von etwa 0.4 bis 1.0 $\mu \mathrm{m}$ infolge der ditektenand werzögerten Bildung dor hydratisierten Elek tonen bis zu $50 \mu \mathrm{s}$ lang ab, um dann infolge
Reaktion (2) (bei allen bisherigen Versuchen im Stahlautoklaven war der in Bezug auf die Elektronen in zweiter Ordnung ablaufenden Reaktion (2) eine Reaktion erster Ordnung mit einer nicht definierten Verunreinigung überlagert) innerhalb $1 \mathrm{~ms}$ wieder auf den Wert der unbestrahlten Lösung anzusteigen.

Aus der Wellenlängenabhängigkeit der optischen Dichte der Lösung $\left(\sim \varepsilon \cdot c_{e^{-}}\right)$nach einem Elektronenimpuls ergibt sich das Absorptionsspektrum der hydratisierten Elektronen. Aus der Zeitabhängigkeit der optischen Dichte ergibt sich die Zeitabhängigkeit der Konzentration der Elektronen und damit ihre Reaktionsgeschwindigkeit.

a) Druckabhängigkeit des Absorptionsspektrums

In Abbildung 1 ist die relative optische Dichte der Lösungen, rückextrapoliert auf den Zeitpunkt des Impulsendes, für 1 und 1000 atm Druck gegen die Wellenlänge aufgetragen. Die $1000 \mathrm{~atm}-$ Kurve ist auf die $1 \mathrm{~atm}-K$ urve normiert. Beide Kurvenzüge wurden aus den Meßpunkten nach der Methode der kleinsten Fehlerquadrate unter der Annahme einer Gaußkurve berechnet.

Das Absorptionsmaximum des $1 \mathrm{~atm}$-Spektrums liegt in Übereinstimmung mit Literaturdaten $[8]$ bei $0.72 \mu \mathrm{m}$, das des $1000 \mathrm{~atm}$-Spektrums ist um $200 \pm 50 \AA$ ins Blaue verschoben. Diese Blauverschiebung, die wir auch bei solvatisierten Elektronen in Ammoniak gefunden haben ${ }^{[3]}$ und die der Rotver-

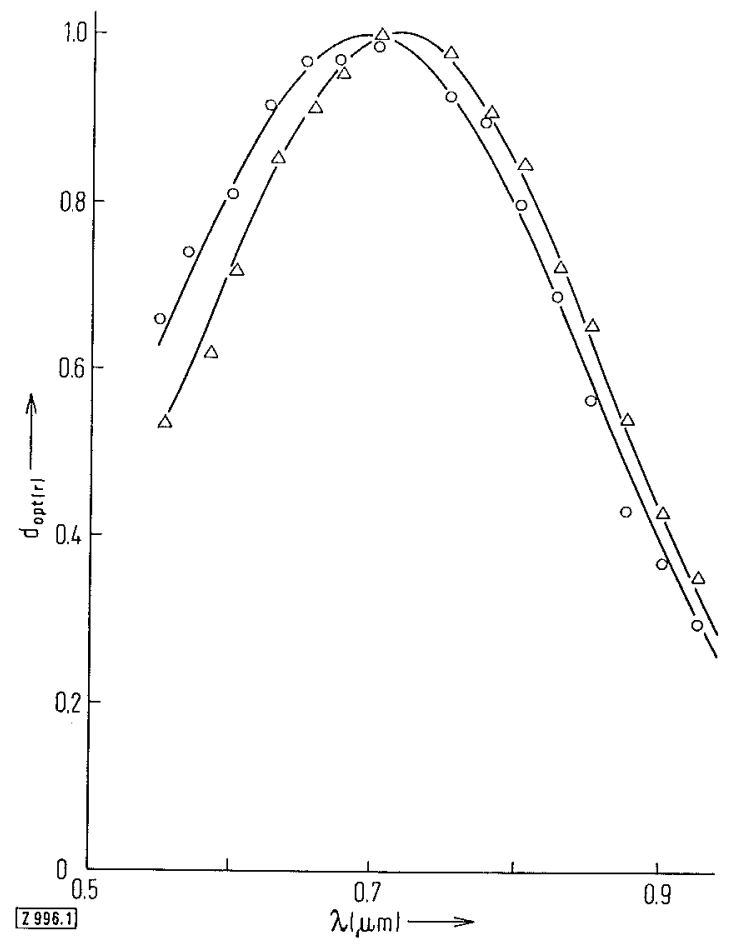

Abb. 1. Absorptionsspektrum hydratisierter Elektronen bei $24^{\circ} \mathrm{C}$ und 1 atni $(\Delta)$ bzw. 1000 atm $(0)$. Die eingezeichneten Meßpunkte sind Mittelwerte aus je 5 Einzelmessungen.

schiebung der Spektren bei Temperaturerhöhung $[1,3,4,8]$ gegenüber gestellt werden muß, läßt sich nach dem Hohlraummodell van lortuer [101 durch Kompression des von den gelösten EJektronen kingenommenen freien Raumes deuten.

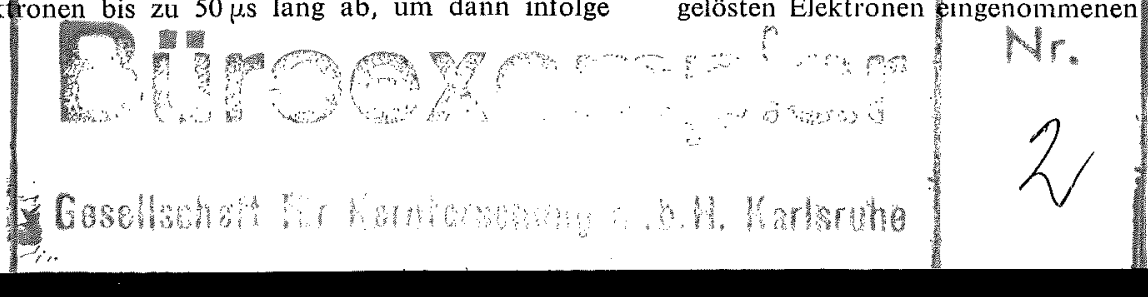


Das Produkt aus $\varepsilon_{\max }$ und $c_{e^{-}}$ist nach Abbildung 1 innerhalb der Fehlergrenze druckunabhängig. Da nach Untersuchungen von Hencz et al. [11], die die Ausbeute stabiler Radiolyseprodukte bei der $\gamma$-Bestrahlung wäßriger Lösungen unter Druck studierten, die Strahlenausbeute hydratisierter Elektronen unabhängig vom Druck ist, folgt, daß auch ihr maximaler Extinktionskoeffizient druckunabhängig ist.

\section{b) Druckabhängigkeit der Reaktionsgeschwindigkeit}

Abbildung 2 zeigt die zeitliche Änderung der relativen optischen Dichte $\left(\sim \mathrm{c}_{\mathrm{e}^{-}}\right)$bei 1 und $1000 \mathrm{~atm}$, jeweils für die Wellenlänge maximaler Absorption. Die Meß3punkte für beide Drücke liegen innerhalb der Fehlergrenze auf einer Kurve, d.h. die Reaktionsgeschwindigkeit oder die mittlere Lebensdauer der hydratisierten Elektronen in Bezug auf Reaktion (2) - und in Bezug auf die Reaktion mit der nicht identifizierten Verunreinigung - ist druckunabhängig, das Aktivierungsvolumen dieser Reaktionen ist verschwindend klein. Die

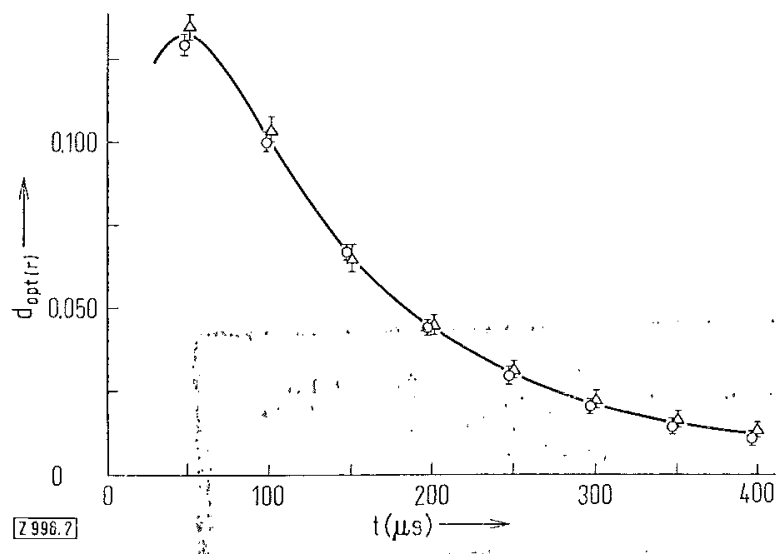

Abb. 2. Zeitlich Abhängigkeit der optischen Dichte einer wäßrigen Lösung nach einem Elektronenstrahlimpuls bei $24^{\circ} \mathrm{C}$ und $1 \mathrm{~atm}(\Delta)$ bzw. $1000 \mathrm{~atm}\left(\mathrm{O}^{\prime}\right)$ Die eingezeichneten Meßpühkte sind Mittelwerte aus je 5 Messungen.

direkten Messungen der Reaktionsgeschwindigkeit unter Druck bestätigen die von Hentz ${ }^{[11]}$ auf indirektem Wege erhaltenen Ergebnisse.

Bei einigen vorläufigen Messungen [12] der sehr langsamen Reaktion zwischen solvatisierten Elektronen und Wasser in flüssigem Ammoniak $\left(20 \% \mathrm{H}_{2} \mathrm{O}, 80 \% \mathrm{NH}_{3}\right.$; Lebensdauer der Elektronen in der Größenordnung von $10 \mathrm{~s}$ bis $1 \mathrm{~min}$; Aktivierungsenergie $<3 \mathrm{kcal} / \mathrm{mol}$ ) finden wir bei Druckerhöhung um $1000 \mathrm{~atm}$ eine Beschleunigung auf das Drei- bis Vierfache, entsprechend einem Aktivierungsvolumen von etwa $-25 \mathrm{ml} / \mathrm{mol}$. Die verschiedene Druckabhängigkeit der Reaktionsgeschwindigkeit in Wasser und Ammoniak spiegelt den unterschiedlichen Raumbedarf der solvatisierten Elektronen in den beiden Lösungsmitteln wieder (in Wasser $<20 \mathrm{ml} / \mathrm{mol}$, in Ammoniak 60 bis $90 \mathrm{ml} / \mathrm{mol}$ ).

Eingegangen am 12. Mai 1969

[Z 996]

[*] Prof, Dr. U, Schindewolf, Dipl.-Phys. H. Kohrmann und Dipl.-Phys. G. Lang

Institut für Kernverfahrenstechnik der Universität und des Kernforschungszentrums

75 Karlsruhe, Postfach 3640

[**] Diese Arbeit wurde von der Deutschen Forschungsgemeinschaft und dem Fonds der Chemischen Industrie unterstützt. Wir danken dem Institut für Strahlentechnologie der Bundesanstalt für Lebensmittelfrischhaltung für die Bereitstellung und Herrn $M$. Rudolf für die Bedienung des Linearbeschleunigers. [1] Zusammenfassung: U. Schindewolf, Angew. Chem. 80, 165 (1968); Angew. Chem. internat. Edit. 7, 190 (1968); vgl. auch dort zitierte Literatur.

[2] U. Schindewolf, K. W. Böddeker u. R. Vogelsgesang, Ber. Bunsenges, physik. Chem. 70, 1161 (1966).

[3] U. Schindewolf, Angew. Chem. 79, 585 (1967); Angew. Chem. internat. Edit. 6, 575 (1967).

[4] R. Vogetsgesang; Diśsertation, Universität Karlsruhe 1969.

[5] K. W. Böddeker, G. Lang u. U. Schindewolf, unveröffentlicht.

[6] $U$ : Schindewolf, G. Lang u. $K$. "W, Böddeker, Z, physik. Chem. N. F. 1969, im Drúck.

[7] U. Schindewolf, R. Vogèlsgesang u. $K, W$. Böddeker, Angew. Chem. 79, 1064 (1967); Angew. Chem. internat. Edit. 6, 1076 (1967); K. W. Böddeker, G. Lang u. U. Schindewolf, Angew. Chem. 81, 118 (1969); Angew. Chem. internat. Edit. 8,138 (1969). [8] Solvated Electron. Advances Chem. Ser, 50, 1965.

[9] U. Schindewolf, G. Lang u. H. Kollinan, Chemie-Ing.-Techn., im Druck.

[10] J. Jortner, J. chem. Physics 30, 839 (1959); Radiat. Res. Suppl. 4, 24 (1964); J. Jorther, S. A. Rice u, E. G. Wilson in G. Lepoutre u. M.J. Sienko: Solutions Métal-Ammoniac. Benjamin, New York 1964, S. 222.

[11] R. R. Hentz, Farhataziz u. D. J. Milner, J. chem. Physics 47,4865 (1967).

[12] U. Schindewolf u, $R$. Olinger, Vortrag auf der 68. Bunsentagung, Frankfurt 1969. 


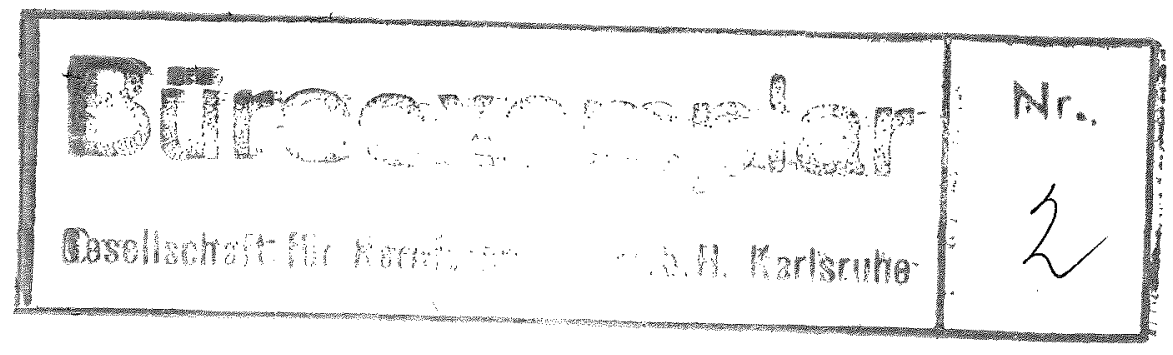

\section{Anordnung zur Impulsradiolyse bei hohem Druck}

\section{Prof. Dr. U. Schindewolf, Dipl.-Phys. G. Lang und Dipl.-Phys. H. Kohrmann}

Institut für Kernverfahrenstechnik der Universität und des Kernforschungszentrums Karlsruhe

Unsere früheren Untersuchungen über das Verhalten von stabilen Metall-Ammoniak-Löstungen unter hohem Druck zeigten, daß die physikalischen und chemischen Eigensohaften der durch Dissoziation des gelösten Metalls entstehenden solvatisierten Elektronen stärker vom Druck abhängen als die Eigenschaften anderer in Ammoniak gelöster Stoffe (Literaturangaben vgl. [1]). Diese hohe Druckabhängigkeit ist bedingt durch den großen Volumenbedarf der solvatisierten Elektronen (60 bis $90 \mathrm{ml} / \mathrm{mol}$ ). Entspre-

[1] U. Schindewolf, H. Kohrmann u. G. Lang, Angew. Chem., $81,496[1969]$ chende Druckuntersuchungen an den kurzlebigen hydratisierten, d. h. in Wasser gelösten Elelktronen (Lebensdaner $<1 \mathrm{~ms}$ ), konnten bisher nicht durchgeführt werden, da diese Untersuchungen die Erzeugung der hydratisierten Elektronen in Kombination mit schnell ansprechenden Nachweismethoden unter Druck voraussetzen. Im folgenden wird eine Apparatur beschrieben, die die Untersuchung des Absorptionsspektrums und der Reaktionsgeschwindigkeit impulsradiolytisch erzeugter hydratisierter Elektronen unter Druck bis zu 1000 at ermöglicht. Sio setzt sich, wie Abb. 1 zeigt, aus dem Hochdrucksystem, dem optischen System und dem Linearbeschleuniger zusammen. 


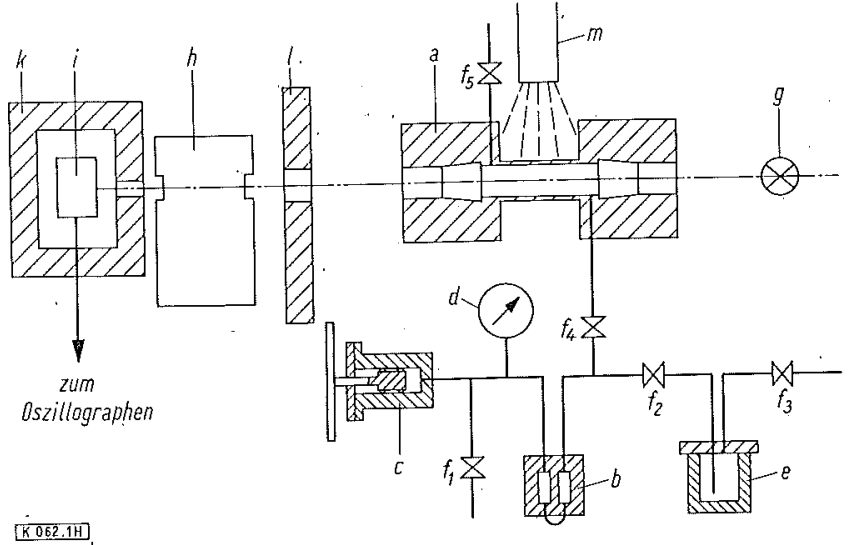

Abb. 1. Anordnung für impulsradiolytische Untersuchungen unter Druck.

$a$ Hochdruck-Küvette, $b$ Druckvermittler, $a$ Druckgenerator, d Manometer, e Flüssigkeitsvorratsautoklaven, $f_{1}$ bis $f_{5}$ Ventile, $g$ Lampe, $h$ Monochromator, $i$ Lichtdetelztor, $k$ und $l$ Blei-Abschirmung, in Linearbeschleuniger.

\section{Das Hochdrucksystem}

Das Hochdrucksystem besteht aus der Hochdruckküvette $a^{1)}$, dem Druckvermittler $b^{11}$, dem Druckgenerator $c^{2)}$, dem Manometer $d^{3}$ und dem Flüssigkeitsvorratsautoklaven $e$. Diese aus VA-Stahl gefertigten Teile sind über Hochdruckkapillaren 2) miteinander verbunden. Die nach außen führenden Ventile ${ }^{2} f_{1}, f_{3}$ und $f_{5}$ dienen zum Reinigen, Evalkuieren und Füllen des Systems.

Im Druckgenerator wird der Druck durch Eindrücken eines zylinderförmigen Stempels in einen mit Wasser gefüllten Zylinder (Volumen $10 \mathrm{ml}$ ) erzengt. Um zu vermeiden, daß bei der Druckerhöhung das mit Schmieröl verumreinigte Wasser aus der Pumpe in die Küvette gedrückt, wird, ist zwischen beide der Druckvermittier $b$ mit einem Innenvolumen von $10 \mathrm{ml}$ geschaltet.

Bei der Konstruktion der Hochdruckkiüvette (Abb. 2) mußte bezüglich der Wandstärke ein Kompromiß geschlossen werden. Die Wände mußten einerseits so stark sein, daß sie dem geforderten Druck von wenigstens 1000 at standhalten, sie mußten aber andererseits so dünn gehalten werden, daß

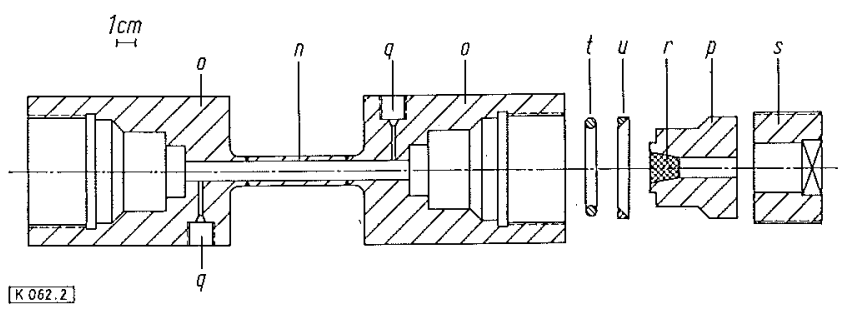

Abb. 2. Schnitt durch die Hochdruck-Kúvette.

n Mittelteil, o Stahlkörper, $p$ Fensterhalterungen, $q$ Kapillaren, $r$ Saphir-Fenster, $s$ Hohlschraube, $t$ Dichtring, $u$ Gleitring.

die die Radiolyse auslösenden Elektronen nicht vollständig abgebremst werden. Die praktische Reichweite von $10-\mathrm{MeV}$ Elelsti'onen in Stahl ist annähernd $5 \mathrm{~g} / \mathrm{cm}^{2} \triangleq 6 \mathrm{~mm}$. Um zu gewährleisten, daß die Lösung auf ihrem ganzen Querschnitt gleichmäßig den Elektronen ausgesetzt ist, wurde die Wandstärke auf 2,5 mm festgelegt. Bei dieser Wandstärke und einer Belastbarkeit von 1000 at darf der innere Durchmesser unter Berücksichtigung einer zweifachen Sicherheit $10 \mathrm{~mm}$ nicht überschreiten.
Da in das dïnnwandige Rohr weder die Fenster noch die Verbindungskapillaren eingesetzt werden können, ist nur der dem Elektronenstrahl ausgesetzte $7 \mathrm{~cm}$ lange Mittelteil $n$ der Hochdruckkzüvette nach den angegebenen Maßen gefertigt. An die beiden Enden sind massive, der Länge nach aufgebohrte V2A-Stahlkörper $o$ angeschweißt, in die die Fensterhalterungen $p$ und Kapillaren $q$ eingeschraubt werden. Die für Licht im Wellenlängenbereich von 0,3 bis $5 \mu \mathrm{m}$ durchlässigen, konischen Saphir-Fenster $r^{4}$ sind in die entsprechend ausgebohrten Halterungen $p$ eingeschliffen, die mit Hohlschrauben $s$ und zugehörigen Dicht- und Gleitringen $t$ und $u$ in die Endstïcke des Autoklaven druckfest eingeschraubt sind. Die konische Form der. Fenster gewährleistet ohne zusätzliche Maßnahmen eine einwandfreie Dichtung zwischen Fenster und Halterung.

Zur Reinigung wird durch das Hochdrucksystem mehrero Stunden überhitzter Wasserdampf geblesen. Die evakuierte Küvette wird über das Ventil $f_{2}$ (Abb. 1) mit der in dem Vorratsautoklaven $e$ unter leichtem Überdruck befindlichen Meßlösung gefüllt.

\section{Das optische System}

Die Hochdruckküvette, die auf einer optischen Bank angebracht ist, befindet sich in Strahlengang eines Spektralphotometers $^{5)}$ (Lampe $g$, Monochromator $h$, Lichtcletektor i). Der Lichtdetektor ${ }^{6}$ ) wandelt Schwankungen des einfallenden Lichtes infolge Änderung der Lichtdurchlässigkeit der bestrahlten Löstung in dazu proportionale elektrische Signale um, die über einen Impedanzwandler auf die Ablenkplatten eines durch den Elektronenbeschleuniger getriggerten Kathodenstrahl-Oszillographen" gegeben werden. Das System wird durch Einschalten einer rotierenden Lochscheibe in den Strahlengang (wechselweise 0 und $100 \%$ Lichtdurchgang) geeicht.

Da die Photozelle geringfügig auch auf die direkte und rückgestreute Bremsstrahlung anspricht, wurde sie allseitig mit einer $5 \mathrm{~cm}$ starken Blei-Schicht $k$ abgeschirmt. Um Verfärbungen des Prismas und der Linsen durch Strahlenschädigtung zu vermeiden, wurde der Monochromator ebenfalls durch eine $5 \mathrm{~cm}$ starke Blei-Sohicht $l$ gegenüber dem Elektronenstrahl abgeschirmt.

\section{Der Elektronenbeschleuniger}

Die Radiolyse der Lösung wird durch energiereiche Elektronen eines Linearbeschleunigers $m^{8)}$ ausgelöst, der bis zu 300 Elektronenimpulse mit einer Impulsdauer von je $5 \mu \mathrm{s}$ liefert. Bei einer Energie von $10 \mathrm{MeV}$ lassen sich Impulsströme bis zu $500 \mathrm{~mA}$ erzielen.

\footnotetext{
1) $a$ und $b$ wurden in der Institutswerkstatt gebaut.

2) High Pressure Equipment Co., Erie/Penn. (USA).

3) Haenni \& Cie. mbH, Bad Cannstatt.

4) Badische Industrie-Edelstein-Gesellschaft mbH, Elzach/ Schwarzwald.

5) Spektralphotometer PMQII, Carl Zeiss, Oberkochen.

6) Photozelle $90 \mathrm{AV}$ bzw. $90 \mathrm{CV}$ für die Wellenlängenbereiche von 0,3 bis $0,65 \mu \mathrm{m}$ bzw. 0,6 bis $1 \mu \mathrm{m}$, Valvo GmbH, Hamburg.

7) Oszillar M214, Siemens AG, Karlsruhe.

8) Elektronen-Linearbeschleuniger V-7703 der Firma Varian Associates, Palo Alto/Cal. (USA). - Wir danken dem Institut für Strahlentechnologie der Bundesanstalt für Lebensmittel. frischhaltung für die Bereitstellung sowie Herrn M. Rudolf für die Bedienung des Linearbeschleunigers.
} 


\section{Empfindlichleeit der Anordnung}

Der Elektronenstrahl ist im Abstand von $3 \mathrm{~m}$ vom Beschleunigungssystem, der aus apparativen Gründen eingehalten werden $m u ß$, auf etwa $100 \mathrm{~cm}^{2}$ aufgefächert, so daß sich am Ort des Meßsystems eine Impulsstromdichte von maximal $5 \mathrm{~mA} / \mathrm{cm}^{2}=1,5 \cdot 10^{11}$ Elektronen $/ \mathrm{cm}^{2}$ ergibt. Die energiereichen Elektronen geben in Wasser infolge Ionisation und Anregung je Zentimeter Laufweg eine Energie von etwa $1 \mathrm{MeV}$ ab. Bei einer Ausbeute von 2,5 solvatisierten Elektronen je $100 \mathrm{eV}$ absorbierter Energie werden also je Elektronenimpuls etwa $3,75 \cdot 10^{15}$ solvatisierte Elektronen je Milliliter gebildet. Da unter den gewählten experimentellen Bedingungen die mittlere Lebensdauer der Elektronen $(150 \mu \mathrm{s})$ groß ist gegenüber der Impulsdauer, kann nach Impulsende mit einer Maximalkonzentration solvatisierter Eleltronen von $6 \cdot 10^{-6} \mathrm{~mol} / \mathrm{l}$ gerechnet werden. Die maximale Änderung der optischen Dichte $D$ der Lösung infolge der Bildung solvatisierter Elektronen

$D=\varepsilon \cdot c \cdot d=\lg \frac{I_{0}}{I}$

( $\varepsilon$ molarer Extinktionskoeffizient $=1,5 \cdot 10^{4} \mathrm{l} / \mathrm{mol} \cdot \mathrm{cm}$ beim Absorptionsmaximum $7200 \AA$; c Konzentration der hydra. tisierten Elektronen; $d$ Lichtweg $=7 \mathrm{~cm}, I_{0}$ Lichtdurchlässigkeit der unbestrahlten Lösung, $I$ Lichtdurchlässigkeit der bestrahlten Lösung) ist damit im Absorptionsmaximum der solvatisierten Elektronen etwa 0,63, so daß nach dem Beerschen Gesetz die Lichtclurchlässigkeit der bestrahlten Lösung nach Impulsende um maximal 75\% abnehmen kann. Da noch Änderungen der Lichtdurchlässiglkeit von $1 \%$ gut nachgewie- sen werden kömmen, bereitet der photometrische Nachweis der solvatisierten Elektronen mit der beschriebenen Anordnung auch bei einer hundertfach kleineren Konzentration $\left(10^{-8}\right.$ bis $\left.10^{-7} \mathrm{~mol} / \mathrm{l}\right)$ keine Schwierigketi.

Die beschriebene Anordnung wurde getestet durch die Untersuchung der Druckabhängigkeit des Absorptionsspektrums und der Lebensdauer hydratisierter Elektronen [1]. Das Spektrum, dessen Absorptionsmaximum unter Normalbedingungen bei $0,72 \mu \mathrm{m}$ liegt, wird bei Drucksteigerung auf 1000 at um $200 \pm 50 \AA$ zu kiurzeren Wellenlängen verschoben. Diese Verschiebung kann ebenso wie die des Spektrums gelöster Elektronen in Ammoniak (Absorptionsmaximum bei 1,5 bis $1,8 \mu \mathrm{m}$, Versehiebung je nach Temperatur 500 bis $1000 \AA / 1000$ at) durch eine Kompression der von den Elektronen besetzten Lösungsmittelhohlräume gedeutet werden. Die Lebensdauer der hydratisierten Elektronen, die durch die Reaktion mit Wasser

$$
2 \mathrm{e}^{-}+2 \mathrm{H}_{2} \mathrm{O} \rightarrow \mathrm{H}_{2}+2 \mathrm{OH}^{-}
$$

begrenzt ist (Geschwindigkeitskonstante: $10^{10} \mathrm{l} / \mathrm{mol} \cdot \mathrm{s}$ ), wird vom Druck nicht beeinflußt. Im Gegensatz dazu ergibt sich für die gleiche Realktion in flïssigem Ammonials bei Drucksteigerung um 1000 at eine Beschleunigung auf das Drei- bis Vierfache. Diese unterschiedliche Druckabbängig. keit der Reaktionsgeschwindigkeit oder des daraus folgenden Aktivierungsvolumens der Reaktionen ist auf den unterschiedlichen Raumbedarf der solvatisierten Elektronen in Wasser (<23 ml/mol) und in Ammonialk (60 bis $90 \mathrm{ml} / \mathrm{mol}$ ) zurückzuführen.

Eingegangen am 19. Nai 1969 [K 062] 